\title{
Intuitive Dualism and Afterlife Beliefs: A Cross-Cultural Study
}

Authors: H. Clark Barrett ${ }^{\mathrm{a}, \mathrm{b},}$, , Alexander Bolyanatz ${ }^{\mathrm{c}}$, Tanya Broesch ${ }^{\mathrm{d}, 1}$, Emma Cohen $^{\mathrm{e}, \mathrm{f}}$, Peggy Froerer $^{\mathrm{g}}$, Martin Kanovsky ${ }^{\mathrm{h}}$, Mariah G. Schug ${ }^{\mathrm{i}, 2}$, and Stephen Laurence ${ }^{\mathrm{j}, \mathrm{k}}$

\author{
Affiliations: \\ ${ }^{a}$ Department of Anthropology, University of California, Los Angeles, CA 90095-1553, USA \\ ${ }^{\mathrm{b}}$ Center for Behavior, Evolution, and Culture, University of California, Los Angeles, CA 90095- \\ 1553, USA \\ ${ }^{c}$ Social Sciences Subdivision, College of DuPage, Glen Ellyn, IL 60137-6599, USA \\ ${ }^{d}$ Department of Psychology, Emory University, Atlanta, Georgia, 30322, USA \\ ${ }^{\mathrm{e}}$ Institute of Cognitive and Evolutionary Anthropology, University of Oxford, 64 Banbury Road, \\ Oxford OX2 6PN, UK \\ ${ }^{\mathrm{f}}$ Wadham College, Parks Road, Oxford, OX1 3PN, UK \\ ${ }^{\mathrm{g}}$ Department of Social Sciences, Media and Communications, Brunel University London, \\ London, UK \\ ${ }^{\mathrm{h}}$ Institute of Social Anthropology, FSEV, Comenius University, 82005 Bratislava 25, Slovakia. \\ ${ }^{i}$ Department of Psychology, Wesleyan University, 207 High St., Middletown, CT 06459, USA \\ ${ }^{j}$ Department of Philosophy, University of Sheffield, 45 Victoria Street, Sheffield, S3 7QB, UK \\ ${ }^{k}$ Hang Seng Centre for Cognitive Studies, University of Sheffield, Sheffield, S3 7QB, UK
}

*Correspondence to: H. Clark Barrett, email: hclarkbarrett@gmail.com

\section{Word count: 8483 words (main text)}

Keywords: [6 max]: mind-body dualism; afterlife beliefs; culture and cognition; explicit versus implicit reasoning; intuitive dualism; intuitive materialism

\footnotetext{
${ }^{1}$ Current affiliation: Department of Psychology, Simon Fraser University, 8888 University Drive, Burnaby, BC, V5A 1S6, Canada

${ }^{2}$ Current affiliation: Department of Psychology, Widener University, One University Place, Chester, PA 19013, USA
} 


\begin{abstract}
It is widely held that intuitive dualism - an implicit default mode of thought that takes minds to be separable from bodies and capable of independent existence-is a human universal. Among the findings taken to support universal intuitive dualism is a pattern of evidence in which "psychological" traits (knowledge, desires) are judged more likely to continue after death than bodily or "biological" traits (perceptual, physiological and bodily states). Here we present crosscultural evidence from six study populations, including non-western societies with diverse belief systems, that shows that while this pattern exists, the overall pattern of responses nonetheless does not support intuitive dualism in afterlife beliefs. Most responses of most participants across all cultures tested were not dualist. While our sample is in no way intended to capture the full range of human societies and afterlife beliefs, it captures a far broader range of cultures than in any prior study, and thus puts the case for afterlife beliefs as evidence for universal intuitive dualism to a strong test. Based on these findings we suggest that while dualist thinking is a possible mode of thought enabled by evolved human psychology, such thinking does not constitute a default mode of thought. Rather, our data support what we will call intuitive materialism - the view that the underlying intuitive systems for reasoning about minds and death produce as a default judgements that mental states cease to exist with bodily death.
\end{abstract}

\title{
1. Introduction
}

In one form or another, explicit belief in supernatural entities and an afterlife is culturally widespread. For example, a 2010 survey found that $84 \%$ of the world's population identify with a religious group and many of the unaffiliated nonetheless hold religious or spiritual beliefs (Pew Research Center, 2012), while a 2008 survey found that more than $90 \%$ of people in the United States believed in God and 74\% believe in life after death (Pew Research Center, 2008). These explicit beliefs in supernatural entities and life after death are widely thought to be supported by an intuitive dualism - a natural default mode of thought that takes minds to be separable from bodies and capable of independent existence.

Many contemporary accounts hold that intuitive dualism arises as an inevitable, though accidental, consequence of the fact that humans possess independent systems for thinking about bodies and minds: "intuitive physics" mediates our thinking about physical objects as such, while "intuitive psychology", or "theory of mind" underlies our thinking about mental entities. Because 
humans are both physical and psychological entities, both systems can be applied to thinking about humans, but each generates different kinds of inferences: intuitive physics generates inferences about humans as physical objects, and intuitive psychology generates inferences about humans as intentional agents (Carey, 2009).

An initial way of understanding the independence of the physical and psychological inference systems is embodied by what we will call the parallel systems account. On this account, physical and psychological inference systems produce what Keil (1989) calls two distinct "modes of construal," two ways we can think about humans and other animals: we can sometimes think of them as physical objects, and at other times as psychological agents, flipping back and forth between these modes like different ways of seeing a Necker cube. For example, we might use our intuitive physics to predict what will happen physically when two players jump for a ball and collide, and our intuitive psychology to predict the emotional responses of each player to the collision.

The parallel systems account implies that people possess distinct ways of thinking about organisms — as agents, and as objects — and that the judgments made in accordance with these different ways of thinking about organisms are made by independent systems. As a result, it allows for the possibility of conceiving of minds and bodies as distinct and capable of independent existence. But it does not mandate that people think of those aspects of organisms as distinct and separable facets of agents. Nonetheless, some see the separability of body and mind involved in intuitive dualism as a natural consequence of the parallel systems account. For example, Paul Bloom (2007) writes:

It is not controversial that young children naturally make sense of physical entities in different terms than psychological entities: naïve physics is different from naïve psychology. The claim explored here is considerably stronger. It is the idea that we think of bodies and souls as distinct; we implicitly endorse a strong substance dualism of the 
sort defended by philosophers like Plato and Descartes. Under one variant of this account, our dualism is a natural by-product of the fact that we have two distinct cognitive systems, one for dealing with material objects, the other for social entities. These systems have incommensurable outputs. Hence dualism emerges as an evolutionary accident (Bloom, 2007, p. 149).

Since intuitive dualism is closely linked to the parallel systems account, the two accounts have many overlapping predictions. For example, both accounts predict that we can sometimes think about humans as physical bodies, e.g., to think about their mechanical response to forces, and sometimes as intentional agents, e.g., to think about their emotional responses to events. However, while the intuitive dualism account predicts all or most of what the parallel systems account does, it also involves additional predictions arising from the fact that intuitive dualism treats minds and bodies as distinct, separable, and capable of independent existence. For example, intuitive dualism predicts that not only is it possible for humans to consider minds and bodies to be distinct and capable of independent existence, but that this way of thinking of minds and bodies should be the natural default mode of thought for humans. ${ }^{3}$ And unlike the simple parallel systems account, intuitive dualism predicts that dualist afterlife beliefs where the soul (mind) can survive the death of the body should be widespread and habitually activated when thinking about death.

An important question raised by Bloom's account of dualism is whether intuitive dualism is true of everyone - a hypothesis that we will call universal intuitive dualism - or whether it might only be true of those in certain cultural contexts - a hypothesis that we label culturally variable intuitive dualism. Both possibilities are consistent with the idea that "dualism emerges as an evolutionary accident," as Bloom puts it. In the non-universalist account, intuitive dualism

\footnotetext{
${ }^{3}$ It is this last claim that is what makes intuitive dualism distinctive, since all the theories we discuss, ranging from intuitive dualism through intuitive materialism all hold that it is possible for humans to consider minds and bodies to be distinct and capable of independent existence.
} 
might be thought of as a kind of "cultural attractor," easy for the human mind to entertain in light of the parallel systems account, but acquired via cultural transmission rather than emerging directly from the operation of evolved psychology (Sperber, 1996).

The second possibility, universal intuitive dualism, is that intuitive dualism is an inevitable byproduct of the parallel systems of intuitive physics and intuitive psychology. On this view, we cannot help but think of minds and bodies as distinct, even if we might think this "implicitly," in Bloom's terms, rather than as an explicit, conscious thought. This view predicts that we should be able to find evidence of intuitive dualism everywhere. For example, even people who explicitly call themselves materialists and anti-dualists should implicitly reason in accordance with intuitive dualism.

Contrasting with both of these versions of intuitive dualism is a third view, intuitive materialism, the view that the underlying intuitive systems for reasoning about minds and death produce as a default judgement that mental states cease to exist with bodily death. The core idea of this view stems from the cessation of agency hypothesis (Barrett and Behne 2005), which posits that evolved mechanisms for reasoning about agency, or the goal-directed actions of living things, cease to be applied to organisms that are dead. These mechanisms attribute minds to living physical bodies that meet certain conditions of agency. A dead human or animal is recategorized as no longer an agent, and therefore psychological states such as seeing or knowing are no longer attributed to the body of that individual. Thus, the natural default pattern of reasoning should be to treat the minds of dead individuals as ceasing with death.

According to this hypothesis, individuals need not have any afterlife beliefs at all; indeed, it predicts that some people will simply view death as extinction of the mind and, following thereafter, disintegration of the body. At the same time, however intuitive materialism does not 
entail that it is impossible for people to entertain thoughts about minds existing independent of their bodies (just as, for example, intuitive physics does not entail that we can't think about quantum mechanics or particle/wave duality). Given the parallel systems account, organisms can be thought of both as physical bodies and as agents. And given the agency tracking mechanisms, dead organisms are construed as bodies without agency. However, nothing in the account precludes the possibility that a mind might continue to exist in a disembodied state or that a mind might come to inhabit a different body. ${ }^{4}$ Just as intuitive dualism is compatible with explicit materialism, so intuitive materialism is compatible with explicit dualism. However, intuitive materialism suggests that when people do have afterlife beliefs of these types, they are a kind of epistemic overlay that floats free of the underlying implicit agency tracking system, because that system is not designed to entertain representations of disembodied minds. Accordingly, where dualist beliefs exist, such beliefs should be explicitly held beliefs, and are likely to co-exist with contrary intuitions regarding death generated by our evolved agency tracking systems. We will return to this idea below.

Our primary goal in this paper is to examine these three competing hypotheses using data drawn from a range of different cultures, including non-western societies with diverse belief systems. Intuitive materialism predicts that no one will have an implicit default mode of thought that takes minds to be separable from bodies and capable of independent existence- - though many may have an explicit belief in dualism as a result of culturally transmitted beliefs. People who lack intuitive dualism but have explicit dualist beliefs should have contrary inclinations in drawing inferences regarding the existence of minds after death, sometimes governed by

\footnotetext{
${ }^{4}$ Since belief in an afterlife and beliefs that minds and bodies are distinct and capable of independent existence are compatible with even intuitive materialism, intuitive dualism cannot be characterised merely in terms of allowing for the possibility of such thought. Rather, what makes intuitive dualism distinctive is the claim that such modes of thought are the default.
} 
intuitive systems, and other times governed by explicit beliefs. Universal intuitive dualism predicts that everyone, across cultures, should have a natural default mode of thought that takes minds to be separable from bodies and capable of independent existence. This would naturally lead to explicit dualist beliefs as well. And while it is possible to have explicit beliefs that contradict one's intuitive beliefs, one would nonetheless expect that the vast majority of participants' responses would be governed by dualist beliefs. Culturally variable intuitive dualism predicts that many people in many cultural contexts will have an implicit default mode of thought that takes minds to be separable from bodies and capable of independent existence, but that some people in some cultural contexts will fail to have such a default mode of thought. Participants might be expected to respond relatively homogenously within a given cultural context, and for many cultural contexts, particularly those with explicit dualist beliefs, to be dominated by dualist responses.

We will argue that our data constitute a significant challenge for universal intuitive dualism, and while they are consistent with some version of culturally variable intuitive dualism, intuitive materialism clearly provides the best explanation of the data. We see these results supporting claims of earlier work that afterlife beliefs do not, in fact, provide strong support for intuitive dualism (Cohen et al. (2011), Harris (2015), Hodge (2008), and Watson-Jones et al (2017)). In the next section, we briefly review the existing literature on this topic. Then, in the following section, we describe our own study of judgments about death and the afterlife in six societies, which is designed to provide a cross-cultural test of these three competing hypotheses.

\section{Afterlife beliefs as a test case}


Intuitive dualism should be linked to a range of inferential dispositions regarding potential situations where minds might be seen as separable from bodies and capable of independent existence including, amongst others, cases of spirit possession, body-switching, and reincarnation (Cohen et al., 2011; Kuhlmeier, Wynn, \& Bloom 2004: Chudek et al., 2018). But intuitive dualism is often thought to be particularly closely tied to afterlife beliefs, where the mind is taken to continue to exist separately after bodily death. They will be the focus of our study.

A number of previous studies have examined afterlife beliefs. In a study that was described to participants as a study of people's afterlife beliefs, Bering (2002) presented university students in the US with vignettes involving the death of a person. The study demonstrated a differential pattern of extension of properties (states or traits) to individuals before and after death —in particular, some properties (mental or psychological traits) were more likely to continue to be attributed to the individual after he or she dies, than others (e.g., physical or biological traits). This differential pattern has been taken to be indicative of intuitive dualism.

Bering's analysis categorized the traits into six different kinds: biological (e.g., "will he need to drink again?"), psychobiological (“is he still hungry?"), perceptual (“can he see?”), emotional ("is he still angry at his wife?"), desire (“does he want to be alive?"), and epistemic ("is he thinking about his wife?"). He took these categories to vary along the dimension of less psychological (e.g., purely biological traits like drinking) to more psychological (e.g., epistemic traits like belief). Subjects responses were coded in terms of whether they involved a judgment that a given trait, such as thinking about his wife continued to exist following death (we will refer to these as continued-existence judgments) or they involved a judgment that the trait—whether mental or physical — ceased to exist following death (we will refer to these as cessation 
judgments). Based on interviews, subjects were also categorized according to their religious / spiritual beliefs, with "agnostic" and "extinctivist" (life ends at death) being relatively areligious, and "immortalist," "eclectic," and "reincarnationist" being more religious.

Bering found that the percent of cessation judgments ranged from near $100 \%$ for biological and psychobiological traits to less than $30 \%$ for the emotional, desire, and epistemic traits for the religious / spiritual subjects, while for agnostics and extinctivists, cessation judgments were again near 100\% for biological and psychobiological traits and were between $60 \%$ and $80 \%$ for emotional, desire, and epistemic traits. For the explicit afterlife believers, this shows a strong pattern of differential extension of traits, with biological traits ending at death and psychological traits continuing. Even for the professed non-afterlife believers, there was some pattern of differential extension, with psychological traits continuing to be extended after death in over $20 \%$ of the responses.

One potential problem for this study is that by explicitly framing the study as being about afterlife beliefs, it may have encouraged participant responses based on explicit beliefs, rather than implicit intuitive systems. Since Bering's initial study there have been several replications and extensions, across ages, religious backgrounds, and cultures. These later studies did not adopt this explicit framing, and they also found lower levels of continued-existence responses.

Bering and Bjorklund (2004) examined the development of afterlife beliefs in children in the US, in a range of age groups from 4 years old to 12 years old. They found that over the course of development a pattern of differential extension of traits of different kinds emerged. Young children gave more continued-existence responses overall, but also relatively undifferentiated responses, treating mental and biological/physical properties similarly, but by late elementary school and continuing into adulthood, responses became differentiated, with 
significantly more biological/physical traits being judged to end with death (between $80 \%$ and 100\%) than emotional, desire, and epistemic traits being judged to end with death (between 50\% and $70 \%$; though note, importantly, that the modal response was cessation even for psychological traits).

Bering, Hernández Blasi, and Bjorklund (2005) compared afterlife judgments of children age 4 to 12 years in Catholic versus secular schools in Spain, and found the predicted pattern of differential extension of biological versus psychological traits in both groups, but with lower percentages of cessation responses for the psychological traits for the Catholic children than for the secular children. As in Bering and Bjorklund (2004), the 11-12 year olds collectively judged psychological traits to end with death well over 50\% of the time (ranging between $50 \%$ and $80 \%$ for the categories of questions concerning perceptual, emotional, desire, and epistemic traits).

Two additional studies, in two separate cultures, investigated the effect of religious versus non-religious context on afterlife judgments using a priming methodology. Harris and Giménez (2005) elicited afterlife judgments from 7 and 11 year old Spanish children using two different narratives about the death of an elderly person, which were similar except for certain contextual cues. In the "religious" narrative, a priest is present and explains to a character in the story that the deceased person is "with God." In the "secular" narrative, the death of the elderly person is explained by a doctor, and no religious language is used. We will call this a priming paradigm: two versions of a vignette in which everything is held constant (including the details of the death and how it occurred) except for the religious versus secular context. This methodology is useful in that it allows for the examination of the effect of a religious context on responses that can be readily and consistently applied across different societies. We employ a modified form of this methodology in our own study. 
Harris and Giménez (2005) found several patterns. First, in both younger (7 year old) and older (11 year old) children, slightly fewer cessation judgments were made for "mental" versus "bodily" states (e.g., “can he see anything?” versus “do his eyes work?”). The overall number of cessation judgments declined with age. And, there were considerably more continued existence judgments — for both bodily and mental states — in the religious narrative, compared to the secular one. Worth noting here, and relevant to the intuitive dualism hypothesis, is that both bodily and mental states were more likely to be extended to the afterlife in the religious context. Second, in the secular prime condition, the majority of responses from all subjects were cessation responses. While the religious prime greatly reduced the number of cessation responses, subjects from the same religious population, who explicitly profess afterlife beliefs, gave a minority of afterlife-consistent responses in a neutral context. Harris and Giménez conjecture that "two distinct conceptions of death appear to co-exist," a biological and a religious one (2005, p. 143). If so, this would seem to pose a challenge for universal intuitive dualism, which takes dualist responses to be the intuitive default. It is also consistent with intuitive materialism, if the "biological" conception is the intuitive default and the "religious" conception is an explicitly held overlay — much as beliefs about quantum mechanisms can be held explicitly on top of our implicitly non-quantum intuitive physics.

In a study with adults and children among the Vezo of Madagascar, Astuti and Harris (2008) used a priming paradigm similar to that of Harris and Giménez. In the secular or "corpse" story, an individual's illness and death was described in a non-religious, biologically oriented manner. The "tomb" story described the death of a respected elder and described the construction of a tomb after his death. The Vezo profess strong explicit beliefs about the afterlife of ancestors, so the tomb story was designed to prime religious concepts of the afterlife. Following these 
scenarios, subjects were asked a series of questions about the continued existence of bodily and mental states.

The results were similar to those found by Harris and Giménez (2005). Slightly fewer cessation judgments were made for bodily than mental states, and the religious prime reduced the overall number of cessation judgments for both bodily and mental states. As with Harris and Giménez (2005), it is worth noting that the prime affected both bodily and mental state judgments, inconsistent with a strict intuitive dualism account. And the modal response across all conditions, for both children and adults, was a cessation response. Of the seven properties that Harris and Giménez label as bodily properties and the seven properties that they label as mental properties, more than half of responses in all conditions were cessation responses. Thus, the modal response of children and adults, even with the religious prime, was that the property in question ended with the death of the body.

In a study designed to test the cessation of agency hypothesis, Barrett and Behne (2005) interviewed both German children and Shuar children in Ecuador, ages 3 to 5 years, using vignettes about the death of a person and an animal. In the death condition, events leading to the death of the individual were described (e.g., attack by a predator). In the sleep condition, the individual was described as being tired and going to sleep. After each vignette, the same target questions were asked: “Can it move?”, “Can it be afraid?”, “Could it hurt you?”, "If you walked by and made a noise, could it know you were there?" and "Could it move if you touched it?" These questions thus included both "mental" states (e.g., emotion, perception) as well as "bodily" states (e.g., ability to move). By age 5, both Shuar and German children discriminated sleep from death for all five states, extending them to sleeping but not to dead humans and animals. 
One virtue of Barrett and Behne's study is that the questions regarding mental and physiological/physical traits focused on abilities or capacities rather than occurrent states. So rather than asking, for example, "is he still angry at his wife?" as in Bering (2002), Barrett and Behne (2005) asked "Can it be afraid?". This reduces the possibility of cessation answers in light of contingent circumstances - for example, one might think that in the afterlife, specific concerns that one had while alive become inconsequential, and so if someone were angry at their wife while alive, they would no longer be angry after death. But one might think that they nonetheless still had the ability to feel angry or afraid. Framing questions as ability questions thus increases the chances of finding evidence for intuitive dualism if intuitive dualism is correct, and we adopted this practice here.

\section{The present study}

In this study we borrowed elements from the methods of this prior work, particularly Barrett and Behne (2005) and the priming methodologies of Harris and Giménez (2007) and Astuti and Harris (2008). Our goals were twofold. First, we aimed to use a single, common methodology across a wider range of cultures than had previously been tested, to get a better measure of cultural variability and universality in judgments about death and the afterlife. Though two prior studies each examined a single small scale society (Astuti and Harris 2008; Watson-Jones et al. 2017), the majority of prior studies have looked at large-scale societies where major, monotheistic religions predominate; our sample included several smaller-scale societies with a diversity of spiritual belief systems (Note that there exist other multi-site surveys of explicit afterlife beliefs, but these studies target explicitly held beliefs rather than intuitive theories like intuitive dualism (e.g., Atkinson \& Bourrat, 2011).) Second, and related to the first 
goal, we aimed to put the universal intuitive dualism hypothesis to a strong test, by examining afterlife judgments across a diverse sample of societies, and by using a methodology designed to elicit dualist afterlife beliefs if and where they exist, by using ability-based questions as in Barrett and Behne (2005) and by employing the priming methodology of Harris and Giménez (2007) and Astuti and Harris (2008).

Our questions, like those in the previous work that our study is based on, are designed to tap into intuitive inference systems by asking subjects to make productive judgements about novel questions involving sleeping and dead agents, based on an intuitive inference system, rather than simply replying based on a memorized set of facts about sleep, death, or the afterlife. For example, one question asks whether an agent who has died will be able to remember where their house is, a question that participants would likely not have previously entertained. Thus, while our task involves asking explicit questions, as do all standard tasks in this literature, it is designed, like tasks in prior studies, to tap into tap into subjects' intuitive judgements in making productive inferences rather than elicit stored explicit beliefs. ${ }^{5}$ To the extent that these methods are capable of tapping into participants' intuitive judgements, the universal intuitive dualism view would lead us to expect a dualistic pattern of responses. And, since subjects' explicit beliefs in all populations are likely to be dualist, then where responses fail to conform to a dualistic pattern of responses, this cannot be explained either by explicit beliefs or by intuitive dualism, and thus is best explained by an intuitive materialist inference system.

\subsection{Methods}

\footnotetext{
${ }^{5}$ Employing explicit tasks of the type we employ here (vignettes and explicit questions that require participants to productively reason about the domain) is a widely used methodology to tap into intuitive theories in other areas of cognitive science (see, for example, Keil (1989) on intuitive biology and Matan and Carey (1991) on intuitive theories of artifacts).
} 
We used a vignette methodology similar to the studies described above, with two pairs of conditions, one within subjects and one between subjects. Within subjects, individuals heard two vignettes, in counterbalanced order: a Sleep vignette, in which an individual is tired and goes to sleep, and a Death vignette, in which in individual is attacked and killed by a predator. In each vignette the subject was shown a picture of a person, and read a short accompanying vignette.

The Sleep vignette was:

"This man has been working all day and running around. It's night now and he is very tired. He lies down and sleeps. Because he was so tired, he goes straight to sleep and falls into a deep sleep."

The Death vignette was:

"This man has been walking for a long time and is very tired. Suddenly a [named predator-e.g., jaguar] springs out of the woods and attacks the man. The [jaguar] bites him many times on his head and neck, and the man dies. The [jaguar] runs off. The man is dead."

The vignettes were translated into the local language and modified slightly for individual cultural contexts (see Supplementary Materials for more details). For the Death condition, participants were shown a photograph of the predator in question, to enhance the distinction of the predation context from the sleep context in the two within-subjects vignettes.

The paired Sleep / Death conditions are adapted from Barrett and Behne (2005), and have the virtue of contrasting parallel cases in which the agent undergoes a change of state into motionlessness, but in only one of which the individual dies. It thus serves as a kind of manipulation check in which the proportion of cessation responses can be compared for a death and non-death situation. For example, an agent's eventual need for food ceases at death, but not 
upon falling asleep, and so we would expect different answers for this question in the Sleep and Death conditions.

Following each vignette, subjects were asked a series of six target questions, which were identical following both the Sleep and Death vignette:

"If you made a noise, could he hear it?"

"If you touched him, could the man move?"

"Will he eventually need food?"

"Can he feel happy or sad?"6

"Will he be able to remember where his house is later?"

"Does he know what kind of food [X] people eat?" (where " $\mathrm{X}$ " is the name of the participant's local culture, e.g., Shuar, American, etc.)

Between subjects, individuals were randomly assigned to one of two priming conditions: A "No prime" condition, and a "Spirit prime" condition. In the No prime condition, subjects were merely administered the Sleep and Death vignettes as described above. In the Spirit prime condition, subjects were first given a short interview about their views on supernatural agents and the afterlife, modified as appropriate for the local cultural context. Here we call this the "Spirit prime" because for some cultures, such as the Shuar, the questions were about spirits or ghosts; for others, such as Chicago evangelical Christian congregation, the questions were about angels. For example:

"I've heard about the Xs. Can you tell me a little bit about them? Can they know about what people do? Can they feel angry or jealous? Do you believe that Xs are real?" (Where

\footnotetext{
${ }^{6}$ Note that the "Happy" question was not asked in the Marajó Island population; see Supplementary Materials for more details.
} 
"Xs" are spirits, angels, or other locally relevant supernatural entities; see Supplementary Materials for details).

In the Spirit prime condition, following this prime, the Sleep and Death vignettes and target questions were administered identically to the No prime condition, except that in the Death vignette, a brief allusion to a religious ritual associated with death being performed was appended to the end of the vignette (for example, " Some hunters come by later and find the man on the ground. They bring the man back to his village and [the priest / pastor blesses the man] and [he is buried with his relatives / a burial ritual is performed]."). This methodology, modified from Harris and Giménez (2007) and Astuti and Harris (2008), was designed to maximize the possibility that subjects would interpret questions about the afterlife in a spiritual / religious context.

Note that that after initial review of this paper, we conducted a replication in Los Angeles using slightly varied materials from the above, to address concerns raised in review: the agent went to sleep in both conditions (but was killed only in one); the Spirit prime was contrasted with an "Irrelevant" prime instead of no prime; and the use of pictures was made parallel across conditions. These modifications did not significantly change the results (see Supplementary Online Materials for details).

\subsection{Study populations}

We administered our questionnaire to 260 participants across six different study populations, which varied on a variety of dimensions. Some (e.g., Wesleyan students) were from large-scale, Western, industrialized societies, with major religions: WEIRD, in the terminology 
of Henrich et al. (2010). Others (e.g., Shuar) were from small-scale societies with a mix of traditional religious beliefs and beliefs imported from large-scale religions (Christianity).

Some of the characteristics of our study populations are summarized in Figure 1. See Supplementary Materials for fuller descriptions of our study sites. ${ }^{7}$

Table 1. Sample characteristics

\begin{tabular}{|l|l|r|r|r|r|r|r|r|r|}
\hline Population & Country & N & & & & & Age & & \\
\hline & & Total & Female & Male & $\begin{array}{l}\text { Spirit } \\
\text { prime }\end{array}$ & $\begin{array}{l}\text { No } \\
\text { prime }\end{array}$ & Mean & Min & Max \\
\hline $\begin{array}{l}\text { Chicago evangelical } \\
\text { Christian congregation }\end{array}$ & USA & 27 & 17 & 10 & 13 & 14 & 33.9 & 23 & 63 \\
\hline Emory Students & USA & 24 & 19 & 5 & 12 & 12 & 20.5 & 18 & 22 \\
\hline Marajó Islanders & Brazil & 61 & 29 & 32 & 31 & 30 & 28.4 & 14 & 69 \\
\hline Shuar & Ecuador & 35 & 15 & 20 & 17 & 18 & 27.7 & 13 & 58 \\
\hline Storozhnitsa & Ukraine & 48 & 41 & 7 & 24 & 24 & 44.1 & 16 & 79 \\
\hline Wesleyan Students & USA & 65 & 35 & 30 & 31 & 34 & 19.1 & 18 & 22 \\
\hline
\end{tabular}

Figure 1. Map of study sites

\footnotetext{
${ }^{7}$ Data from an additional site in India were excluded from the analysis due to methodological variations (see Supplementary Materials for details).
} 


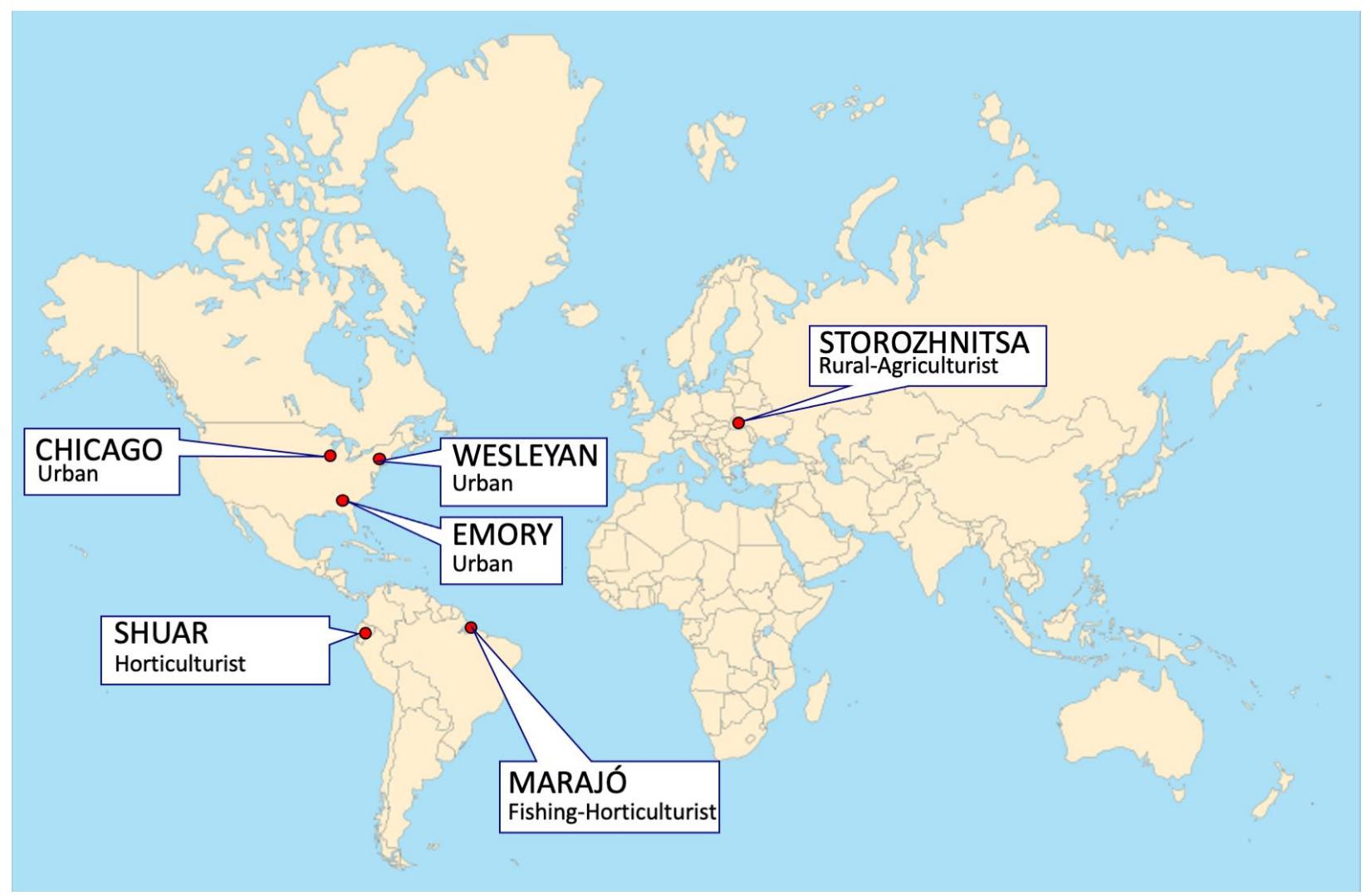

While our sample is in no way intended to capture the full range of human societies and afterlife beliefs, it captures a larger range than prior studies, and thus puts the universal intuitive dualism hypothesis to a strong test.

\section{Results}

\subsection{Analysis 1: Omnibus analysis of all responses across populations and conditions}

Responses of subjects across all study populations and conditions are summarized in Figure 2. In all figures and tables, the outcome variable of interest is cessation responses: these are judgments that the character in the story, after either dying or going to sleep, does not possess the property in question (e.g., need for food, ability to hear a noise). Each individual judgment, therefore, is a binary (yes / no) judgment, and Figure 2 shows the percentage of cessation judgments in each population, pooled by condition. 


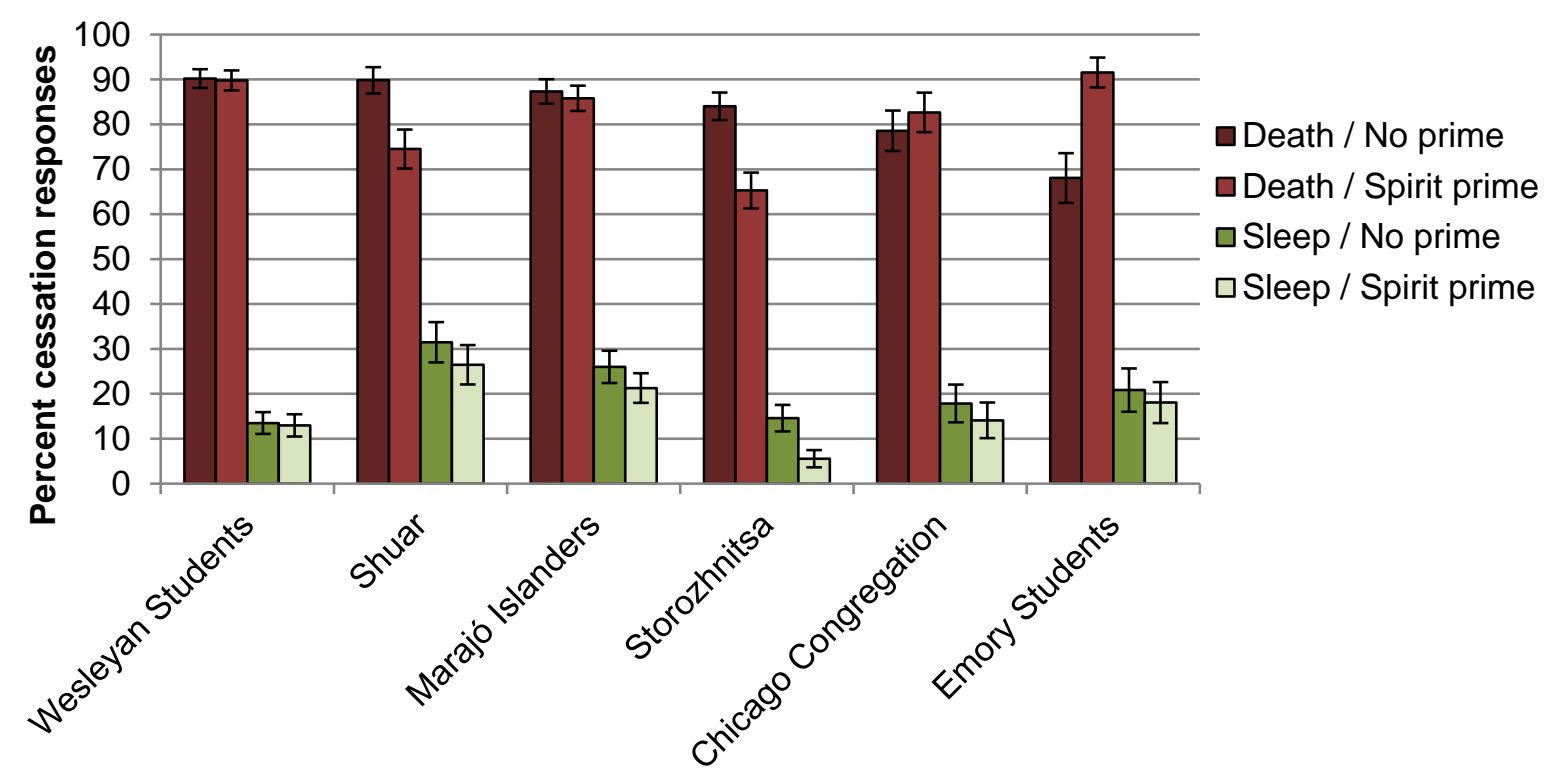

Figure 2. Percent cessation responses by population and condition. Populations are ordered left to right in descending order of percent of cessation responses in the Death / No prime condition.

Error bars represent standard errors.

Inspection of Figure 2 reveals the major patterns in the data. First, the Sleep / Death manipulation had a large effect. Second, the priming manipulation had a relatively small effect, and one whose direction is not uniform across cultures and conditions. Figure 2 pools responses for all properties, both psychological and physiological; we will investigate psychological and physiological traits separately in Analysis 2. While there is some cultural variation in the overall pattern of responses, it is relatively modest.

To examine these effects statistically, we modeled our data using a General Linear Mixed Model, (GLMM), modeling our experimental manipulations as fixed effects (Sleep / Death conditions and Spirit prime / No prime conditions), and variation across subjects and subject populations as random effects, with interaction terms for population-by-fixed effect interactions 
as well as interactions between the Sleep / Death and Spirit prime / No prime condition. The data were modeled using the glmer procedure of the lmer4 package (Bates et al., 2014) in the statistical platform $R$ (R Core Team, 2014). Data were modeled using a binomial (logistic) link function, with cessation judgments as a binary outcome $(1=$ cessation: the individual does not possess the property; $0=$ continued existence: the individual does possess the property).

Parameter estimates of the model are summarized in Table 2.

Table 2. Model parameters for Analysis 1 (omnibus analysis): effects of random and fixed factors on variation in participants' cessation responses. $* * *: p<0.001$.

\begin{tabular}{|l|l|l|l|l|l|l|l|}
\hline Random effects & & & Fixed effects & & & & \\
\hline Factor & Variance & STD & Factor & Estimate & SE & $\boldsymbol{z}$ & $\operatorname{Pr}(>|\mathbf{z}|)$ \\
\hline Subject & 0.696 & 0.834 & & & & & \\
\hline Population & $1.9 \mathrm{e}-10$ & $1.4 \mathrm{e}-5$ & & & & & \\
\hline Population $\mathrm{x}$ Prime & 0.077 & 0.278 & & & & & \\
\hline Population $\mathrm{S}$ Sleep / Death & 0.119 & 0.345 & & & & & \\
\hline & & & Intercept & 1.90 & 0.225 & 8.42 & $<2 \mathrm{e}-16^{* * *}$ \\
\hline & & & Sleep / Death & 3.48 & 0.253 & 13.7 & $<2 \mathrm{e}-16^{* * *}$ \\
\hline & & & Prime & 0.189 & 0.246 & 0.769 & 0.442 \\
\hline & & & Sleep / Death x Prime & 0.164 & 0.214 & 0.769 & 0.442 \\
\hline
\end{tabular}

The patterns visible in Figure 2 are confirmed statistically in the model parameters shown in Table 2. The factor accounting for the largest amount of variation in cessation responses was the Sleep / Death experimental manipulation (significant at $p<.0001$ ). This shows that people in all the populations we sampled showed a strong pattern of differential extension of properties to sleeping versus dead individuals. It also gives a sense of how much variation in responses one might expect even to a relatively straightforward question such as whether a sleeping person could move if touched. Some subjects might, and did, say "no," a perfectly justifiable response. Variation in how the questions were interpreted could also explain some of the variation in responses to the questions about death as well. Importantly, however, cessation responses were 
the modal response in the Death condition in all societies and all conditions, just as continued existence responses were the modal response in the Sleep condition.

Other parameters in the model accounted for much smaller proportions of the overall variation in responses. Among the fixed effects, the main effect of the priming manipulation on participants' cessation responses was relatively small, more than an order of magnitude smaller than the Sleep / Death manipulation. It was not statistically significant, nor was the interaction between the prime and the Sleep / Death contrast. Among random factors, individual variation in responses among subjects accounted for the largest amount of variation, with negligible population variation and small interactions between population and the experimental conditions. This suggests that more of the variation in responses is at the individual level than at the population level.

Because Analysis 1 pooled responses for all trait types, it did not allow us to assess the differential effects of the priming manipulation for different trait types. We assess this in Analysis 2, below.

\subsection{Analysis 2: Comparing physiological and psychological traits in the death condition}

In Analysis 1, all trait types were pooled in order to look at omnibus effects of the Sleep / Death and Priming manipulations. If intuitive dualism is true (in either form, universal or culturally variable) then psychological traits, but not physiological traits, should typically extend into the afterlife. Given this, priming a spiritual or religious conception of death should have a greater effect on psychological traits, which can be thought of as exclusively mental and disembodied (e.g., thoughts, feelings), than on physiological traits, which require a body in order 
to occur (e.g., movement, needing food). Moreover, only the death condition is of particular interest, since intuitive dualism has no bearing on judgments about sleep.

For Analysis 2, then, we fit a GLMM model to the Death condition data, across all the study populations, dividing the six traits into two categories: Physiological (hearing, moving if touched, needing food) and Psychological (feeling happy, remembering, knowing). We treated this distinction between Physiological and Psychological questions (Phys / Psych) as a fixed factor, along with the priming manipulation (Spirit prime / No prime), and included interaction terms for the fixed factors as well as random factors for subject and population. Because there appeared to be a three-way interaction between population, prime, and response to Physiological and Psychological questions, we included a three-way interaction in the model as well.

Figure 3 shows the data from Analysis 2, and Table 3 shows the model parameter estimates for these data. 


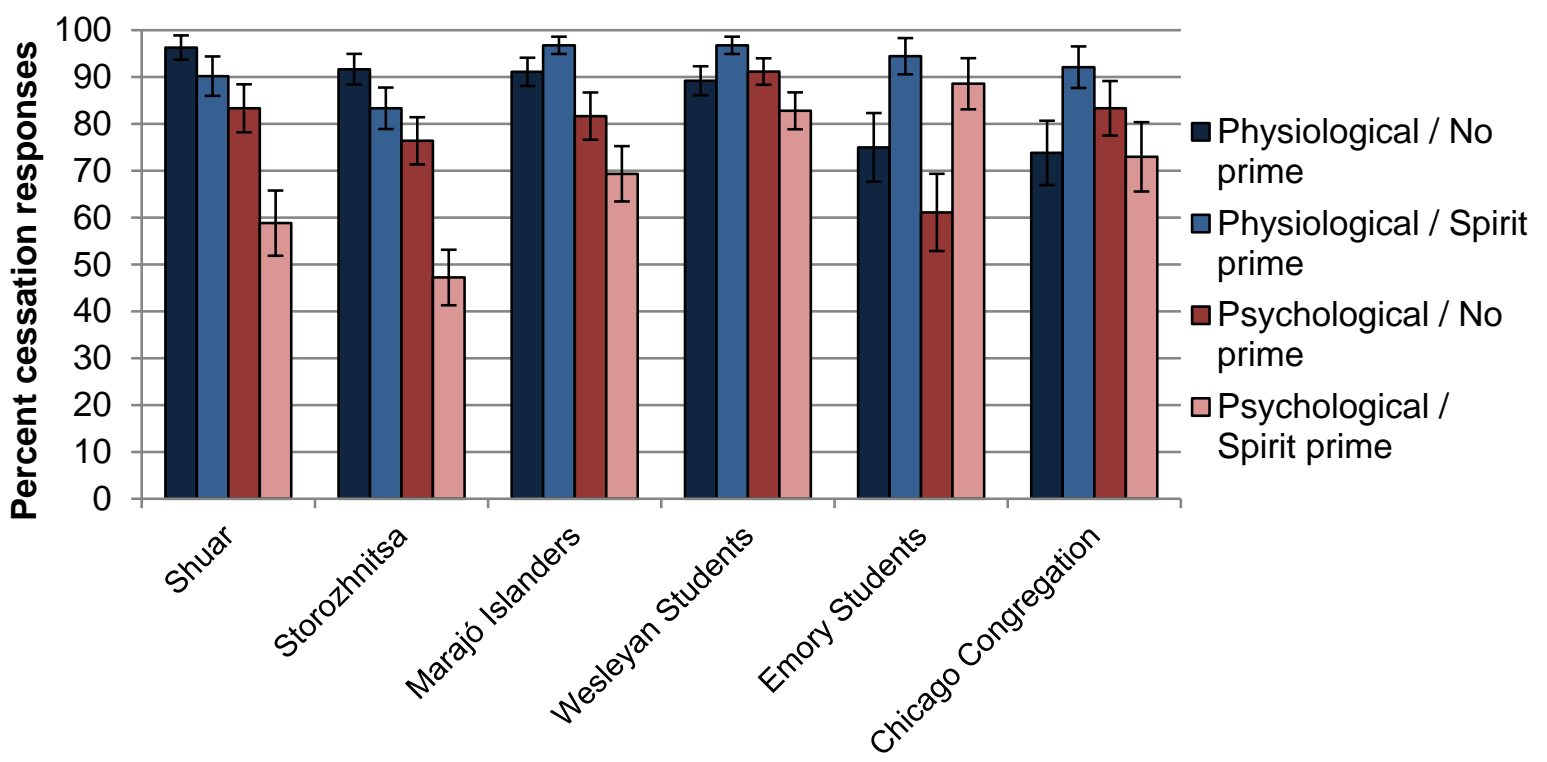

Figure 3. Percent cessation responses in death condition only, by priming condition and trait type. Traits are categorized as physiological (hearing, moving if touched, needing food) or psychological (feeling happy, remembering, knowing). Populations are ordered left to right in descending order of percent cessation responses in the Physiological / No prime condition. Error bars represent standard errors.

Table 3. Model parameters for Analysis 2 (Death condition only): effects of random and fixed factors on variation in participants' cessation responses. ***: $\mathrm{p}<0.001 ; * * \mathrm{p}<0.01 ; *$ : $\mathrm{p}<.05$.

\begin{tabular}{|l|l|l|l|l|l|l|l|}
\hline Random effects & & & Fixed effects & & & & \\
\hline Factor & Var & SD & Factor & Est & SE & $\boldsymbol{z}$ & $\operatorname{Pr}(>|\mathbf{z}|)$ \\
\hline Subject & 8.63 & 2.94 & & & & & \\
\hline Population & $2.34 \mathrm{e}-06$ & 0.0015 & & & & & \\
\hline Population x Prime & $1.70 \mathrm{e}-06$ & 0.0013 & & & & & \\
\hline Population x Psych / Phys & $8.12 \mathrm{e}-07$ & 0.00090 & & & & & \\
\hline $\begin{array}{l}\text { Population x Prime x Psych } \\
\text { IPhys }\end{array}$ & 0.149 & 0.386 & & & & & \\
\hline & & & & & & & \\
\hline & & & Psych / Phys & 0.08 & 0.525 & 7.76 & $8.7 \mathrm{e}-15^{* * *}$ \\
\hline & & & Prime & 0.360 & 2.47 & $0.014^{*}$ \\
\hline & & & Psych / Phys x Prime & 2.24 & 0.610 & 2.04 & $0.042^{*}$ \\
\hline
\end{tabular}


As shown in Table 3, the largest of the fixed effects is the interaction between the Priming condition and the Physiological / Psychological trait factor. Across cultures, in general, the Spirit prime influenced judgments about Psychological traits more than about Physiological traits, generally reducing cessation judgments more for the former than for the latter, as predicted. There was also a main effect of the prime itself, with slightly more cessation judgments overall in the No prime than in the Spirit prime condition. Of the random effects, the largest effect was variation in responses among subjects, which as before was much larger than the amount of variation accounted for by population (which was negligible). The two-way interaction terms were also very small. However, as expected by inspection of the data (Figure 3), there was a modest three-way interaction showing that the effects of the prime on Psychological vs Physiological trait judgments were not uniform across cultures. An effect in which the Spirit prime reduced cessation judgments for Psychological traits was most apparent in the Shuar and Storozhnitsa populations, but reversed in Emory students. Moreover, the prime increased cessation judgments for Physiological traits for Emory students and the Chicago evangelical Christian congregation, which is not predicted by the intuitive dualism hypothesis. This increased the size of the interaction effect. Thus, while the Spirit prime did generally decrease cessation judgments for psychological traits, the overall effects of the prime were mixed: the effects were large only in two cultures, and went in unpredicted directions in some others.

As mentioned, while there was a small main effect of the Psychological / Physiological factor on cessation judgments, the larger pattern to note is that the large majority of judgments were cessation judgments for all traits, across all populations (with the exception of Storozhnitsa for Psychological questions in the Spirit prime condition, where they were around 50\%). 
Analyses 1 and 2 are consistent with the possibility of intuitive dualism in a minority of subjects, albeit with mixed evidence in terms of the specific patterns predicted by the hypothesis. However, both analyses provide strong evidence against the universal intuitive dualism hypothesis, because there were no conditions in any population where a clear majority of responses for psychological traits were continued existence responses.

How were cessation judgments distributed across individual items? It could be that pooling the traits in Analyses 1 and 2 is concealing afterlife responses on some of the traits. Figure 3 shows the percent of cessation responses for each of the Psychological traits-which are the traits that intuitive dualism predicts would be extended to the afterlife — in both the Spirit prime and No prime conditions of the death vignettes, across populations. Even here, there are no cases where the clear majority of responses are continued existence responses. Only five of the bars are under 50\% cessation responses, with error bars encompassing the 50\% line, and none are below $40 \%$. Thus, while there is variation across populations and trait judgments, the majority of judgments were cessation judgments, which are inconsistent not only with intuitive dualism but with afterlife beliefs more generally. 


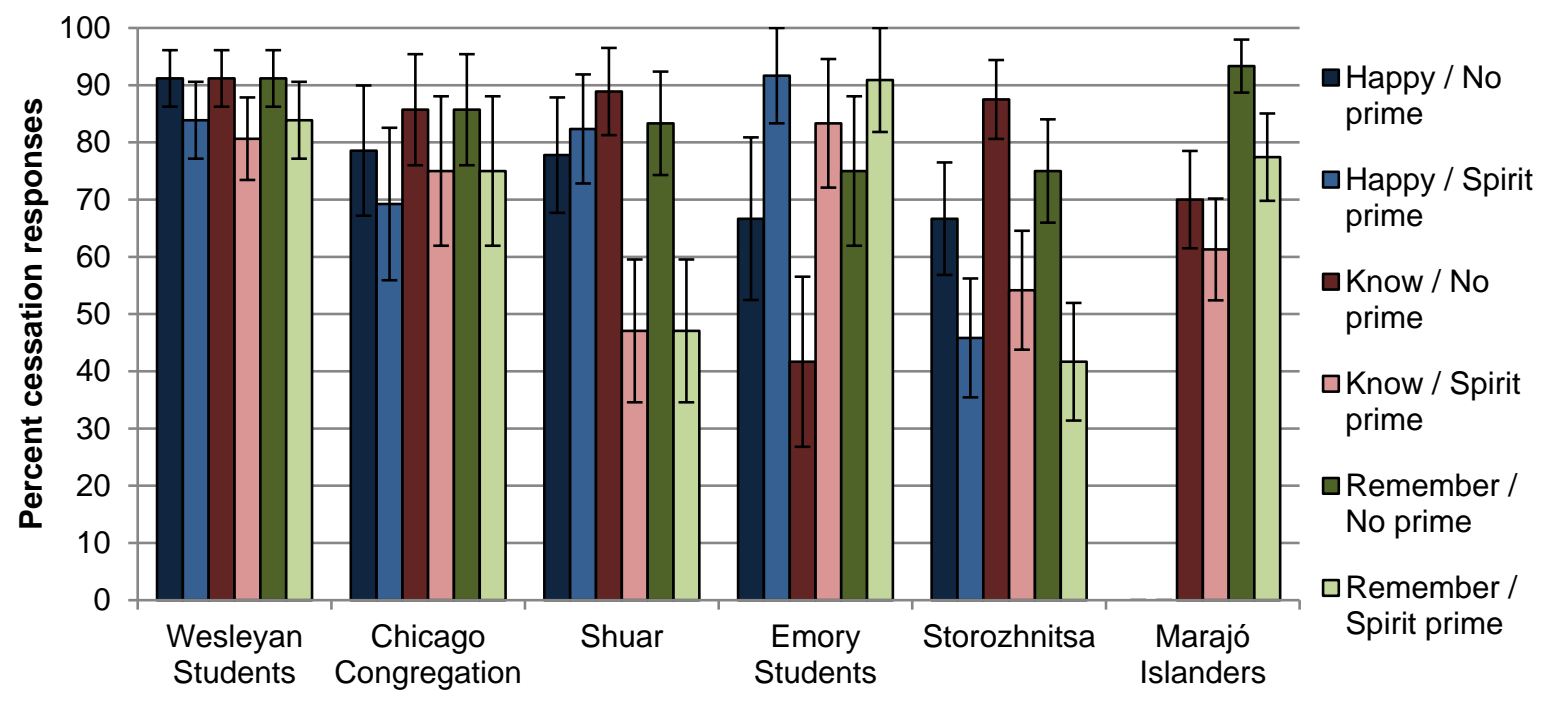

Figure 4. Percent cessation responses for all psychological traits, death condition only. Note that the "happy" question was not asked in the Marajó Island population. Populations are ordered left to right in descending order of percent cessation responses for Happy / No prime. Error bars represent standard errors.

Note also that while some populations, especially Shuar and Storozhnitsa, showed patterns of judgments in response to the priming manipulation consistent with afterlife beliefs, others showed very small effects, with majority responses being cessation responses in most cases. However, while Figures 2 through 4 show that the majority of responses in the death condition were cessation responses, these figures show averages across subjects, and therefore it is possible that most or all subjects gave some afterlife (continued existence) responses, but a small number. To investigate this, we plotted counts of number of individuals who gave specific numbers of continued existence responses, ranging from 0 (all cessation) to 6 (all continued existence) responses (noting that the maximum for Marajó Islanders was 5 responses, because they were not asked the "happy" question). Figure 5 shows histograms of the number of 
individuals providing a given number of continued existence responses (with the Spirit prime and No prime conditions stacked, to show total number of individuals providing a given number of continued existence responses).

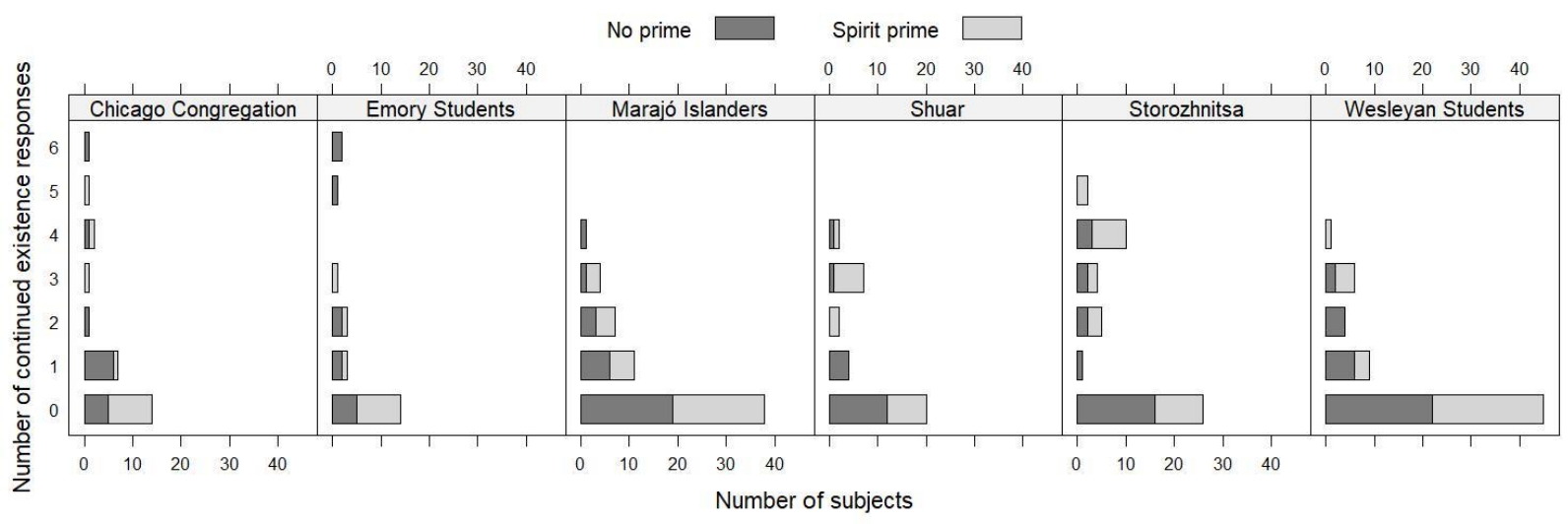

Figure 5. Numbers of individuals giving specific numbers of continued existence responses, by population and priming condition. Note that the maximum number of possible responses per participant is 6 for all populations except in Marajó Islanders, where it is 5.

As shown in Figure 5, the modal response across all six populations was to provide a cessation response for all items - in other words, expression of no continued existence (hence, afterlife) beliefs at all. Table 4 shows the percentage of individuals in each population who gave zero afterlife responses, together with the percentage of individuals in each population who gave consistent afterlife responses to psychological trait questions (feeling happy, remembering, knowing). All of the percentages of zero afterlife responses in Table 4 are over 50\%, indicating that zero-afterlife response patterns were both the modal and majority patterns, in all populations. By contrast, consistent afterlife responses ranged from $6 \%$ to $29 \%$ across 
populations. In the terminology of Bering et al. (2005), the majority of subjects were "consistent cessation theorists," in all six populations.

Table 4. Percent of subjects providing all cessation, all continued existence, and mixed cessation / continued-existence responses to psychological trait questions (feeling happy, remembering, knowing).

\begin{tabular}{|c|c|c|c|}
\hline Population & $\begin{array}{l}\text { All cessation } \\
\text { responses }\end{array}$ & $\begin{array}{l}\text { All continued existence } \\
\text { responses }\end{array}$ & $\begin{array}{l}\text { Mixed } \\
\text { responses }\end{array}$ \\
\hline $\begin{array}{l}\text { Chicago } \\
\text { Congregation }\end{array}$ & $70 \%$ & $19 \%$ & $11 \%$ \\
\hline Emory Students & $63 \%$ & $17 \%$ & $21 \%$ \\
\hline Marajó Islanders & $64 \%$ & $13 \%$ & $23 \%$ \\
\hline Shuar & $60 \%$ & $14 \%$ & $26 \%$ \\
\hline Storozhnitsa & $54 \%$ & $29 \%$ & $17 \%$ \\
\hline Wesleyan Students & $80 \%$ & $6 \%$ & $14 \%$ \\
\hline Total & $66 \%$ & $15 \%$ & $18 \%$ \\
\hline
\end{tabular}

One of the most surprising of our findings was that $70 \%$ of Chicago evangelical Christian congregation - interviewed precisely because of their membership in a Christian congregationgave no afterlife responses at all. Averaged across the congregation, individuals gave between $70 \%$ and $90 \%$ cessation responses, with only a very modest effect of the Spirit prime, which changed responses only by about $10 \%$ on average (see Figure 4 ). We consider possible explanations for these findings in the Discussion.

\section{Discussion}

The clearest conclusion to be drawn from our findings is that there was no support for the universal intuitive dualism hypothesis. In fact, the majority of subjects across all six populations 
we tested showed no evidence of dualism at all, judging that all traits, both physiological and psychological, end at death.

Among the subjects who did provide continued existence responses, there was some evidence of differential extension of psychological traits, compared to physiological traits, to the afterlife. However, the pattern of differential extension was modest, and the effect of the Spirit prime, while statistically significant in the model as a whole, was not uniform across our cultural samples. ${ }^{8}$ Surprisingly, in one population, Emory students, there were more cessation responses in the Spirit prime condition for both physiological and psychological traits than in the no-prime condition (see Figure 3). ${ }^{9}$

How do our results fit with those of earlier studies that have been cited in support of the universal intuitive dualism hypothesis? In spite of how prior results have often been framed, our findings are actually not inconsistent with earlier studies in this area. This work has often been cited in support of universal intuitive dualism, in light of the differential extension of properties into the afterlife, a pattern that we also found. But, the primary result of most previous studies, largely conducted within a single culture, has been broadly consistent with our own findings ranging over six study populations. As Hodge (2008) in particular has argued, though previous

\footnotetext{
${ }^{8}$ The spirit prime that we used was arguably weaker than that used by Harris and Giménez (2005) or Astuti and Harris (2007), who reinforced the prime by prefacing each question with a reminder of this prime. Since intuitive dualism posits an implicit default inference system for reasoning about minds in relation to bodies and the afterlife, this system should be operative with or without the aid of priming. This means that a spirit prime should have little or no impact on continued existence judgements that are the default product of this system. Instead, the prime should be seen as activating an explicit reasoning system affect that is independent of the implicit system posited by intuitive dualism. So, a stronger prime would not make for a stronger test of intuitive dualism. The key finding from the priming manipulation is not the fact that it is that priming can activate an explicit reasoning system to override intuitive judgements produced by an implicit system for reasoning about death (though this is perfectly true). Rather, it is that in the absence of such a prime, cessation responses are overwhelmingly dominant, in strong tension with the existence of a default intuitive dualism.

${ }^{9}$ The effect of the prime for the Emory student population is puzzling and unpredicted. Given the small sample, it requires replication before we can infer that the Emory student population differed in a meaningful way from the other U.S. samples.
} 
studies have not often been seen in these terms, most previous studies have also found that the majority of participant responses have been cessation responses regarding psychological traits just as with physiological / physical traits. For example, as noted above, the priming study of Astuti and Harris (2008) among the Vezo — a society with strong and explicit afterlife beliefsalso found that the modal responses were cessation responses. Astuti and Harris (2008) found that only a minority of subjects provided continued existence responses, much as we did. In other studies, such as Bering (2002), Bering and Bjorklund (2004), and Harris and Giménez (2007), the proportion of cessation judgments across subjects and items has varied, but it is worth noting that no study has yet provided evidence for universal intuitive dualism. Indeed, we would argue that our six-culture study, along with prior studies in individual cultures, point to the same conclusion: intuitive dualism is not a human universal, at least in relation to afterlife beliefs as evidenced by the kind of methodology employed in these studies.

Two of our three hypotheses remain to be considered: the culturally variable intuitive dualism hypothesis and intuitive materialism. While the majority of responses in every culture we looked at were cessation responses, there were continued existence responses in every culture. Overall, $15 \%$ of participants across cultures responded with consistent continuedexistence responses on questions regarding psychological traits, and a further $18 \%$ of participants across cultures responded with a mix of continued-existence responses and cessation responses on questions regarding psychological traits.

One way to account for such responses is to treat them as based on implicit responses of a default intuitive dualism system, in line with the culturally variable intuitive dualism account. The intuitive dualism system can be seen as having been triggered by local cultural conditions, explaining why only a minority of participants in any given study population provided any 
continued existence responses regarding psychological traits. For those participants who did give consistent continued-existence responses to such questions, this might be the direct reflection of the intuitive dualism system. For participants who gave a mix of continued-existence responses and cessation responses to such questions, the explanation would be that for those questions where they gave a cessation response, the response was due to the interaction of intuitive dualism with culturally specific beliefs entailing that one or more of the specific psychological traits our study asked about does not exist in the afterlife. For example, perhaps it was judged that in the afterlife, the concerns of the living would be so remote that in the afterlife one would no longer remember where they had lived or what sorts of foods people eat.

However, this is not the only possible explanation for either consistent continued existence responses or mixed continued existence and cessation responses to questions regarding psychological traits. An alternative type of response would be to deny that any continued existence responses are based on implicit responses of a default intuitive dualism system, and instead take them to be based on explicit afterlife beliefs (see Harris and Giménez 2005 and Astuti and Harris 2008). The only intuitive systems pertaining to death may be the agent tracking systems discussed earlier, which would not attribute any mental states to the dead body. However, alongside these intuitive systems, there may well be explicit beliefs-presumably, in most cases, a culturally transmitted system of explicit afterlife beliefs - that attribute a continued existence to the agent and specific mental states.

The existence of multiple conceptual frames for conceiving of death and its consequences can be thought of along the lines of how we conceive of physics, where an intuitive system exists alongside explicitly held, learned knowledge from physics class. We have an implicit, intuitive system that generates our expectations about how objects will behave - including intuitions 
about their solidity, behavior under gravity, etc. (and which is in many respects "pre-

Newtonian"; McCloskey, 1983). This can coexist alongside explicitly held ideas about relativity, quantum mechanics, etc. (which tell us, for example, that objects are mostly empty space). Each conceptual frame can be employed to reason about objects, but they do not get unified into a single picture. Another example where multiple conceptual frames may be at work is in purposebased reasoning. Kelemen, Rottman, and Seston (2012), for example, found that under speeded processing conditions, scientists who explicitly reject purpose-based explanations of natural phenomena are more likely to endorse such explanations, suggesting that their explicit rejection of such explanations when there are no time restrictions on judgments are governed by one conceptual frame and their endorsement of such explanations in speeded processing is governed by another conceptual frame. Of course, where there are multiple conceptual frames, it is not necessary that each of the frames is equally likely_one may be dominant, or dominant in certain circumstances.

One reason for favouring intuitive materialism over the culturally variable intuitive dualism account in explaining the responses in our study is the gap between the overwhelming dualist responses to explicit general questions about such beliefs in surveys and the responses to questions about a particular case as presented in vignettes in our study. Recall that $84 \%$ of the world's population identify with a religious group (and many of the unaffiliated nonetheless hold religious or spiritual beliefs), more than $90 \%$ of people in the United States believe in God, and $74 \%$ believe in life after death (Pew Research Center, 2008, 2012). By contrast only 15\% of our subjects overall gave consistent continued existence responses to all psychological questions, and only $34 \%$ gave any continued existence responses at all to such questions. In order to explain these results, an intuitive dualist account might try to appeal to multiple conceptual frames—an 
intuitive conceptual frame and an explicit conceptual frame. But since both the conceptual frame in the intuitive dualism system and the explicit conceptual frame hold that minds are separable from bodies, neither conceptual frame has the resources to explain the dominant cessation response that we found. Instead, the best explanation for the dominant cessation response is intuitive materialism. And the best explanation for the glaring difference between responses to explicit general questions about afterlife beliefs in surveys and responses to questions about a particular case as presented in vignettes in our study seems to be that dualist responses to explicit general questions about afterlife beliefs in surveys are governed by explicit dualist beliefs, while the dominant cessation responses are governed by intuitive systems for tracking agency that are posited by intuitive materialism. ${ }^{10}$ This would help to explain why continued existence responses were in the minority even in a study population such as the Chicago evangelical Christian congregation, which has $100 \%$ belief in an afterlife. ${ }^{11}$ Explicit beliefs might also be responsible for continued existence responses in our study, with variation across participants being explained by variation in whether the participant responses were governed by explicit or implicit systems.

Note also that the pattern of decreased cessation responses (increased continued-existence responses) when afterlife beliefs were primed that we found (and which was found in earlier studies) might also be explained through priming activating explicit afterlife beliefs, rather than

\footnotetext{
${ }^{10}$ Could the intuitive dualist account add intuitive materialism as a third conceptual frame in addition to intuitive dualism and explicit (typically dualist) beliefs? In principle it could, but the cost of doing this is that there would no longer be anything distinctive about intuitive dualism. It would no longer be a default intuitive mode of thinking about death and the afterlife, but would instead turn into the weak claim that dualist thought is possible. And this weak claim does not really add anything to intuitive materialism, which already takes dualist thought to be possible.

${ }^{11}$ Another possible explanation in this case could appeal to a subtle form of dualist belief. According to this view, minds (or souls) can continue to exist independent of physical bodies, but they can't function unless united with a physical body. If that is the case, then one might give cessation responses to psychological traits because the mind cannot function until it is re-united with its physical body, when, after Christ's return, the dead will be raised and given new, physical bodies. This seems an entirely possible set of explicit dualist beliefs and may explain why some of the participants from the Chicago evangelical Christian congregation gave cessation responses. However, this very subtle version of dualism seems unlikely to be the implicit default dualist system, and so seems to fit better with intuitive materialism (coupled with explicit, culturally transmitted, dualist beliefs) than with culturally variable intuitive dualism.
} 
triggering an implicit system. Since intuitive dualism posits an implicit default inference system for reasoning about minds in relation to bodies and the afterlife, one might expect this system to be operative with or without the aid of priming. So the main pattern of results that has been taken to support intuitive dualism in afterlife beliefs may be better understood as being driven by activation of explicit afterlife beliefs that are independent of intuitive systems.

\section{Conclusion}

Our data drawn from six study populations, including non-western societies with diverse belief systems, provide strong evidence against the universal intuitive dualism hypothesis in relation to afterlife belief. Both the modal and majority patterns of responses, in all populations, were consistent cessation responses to psychological trait questions. Both the culturally variable intuitive dualism hypothesis and the intuitive dualism hypothesis are consistent with our data, but for the reasons we outlined in the discussion, we think that the latter is more likely.

What is universal, we suggest, are the parallel systems of intuitive physics and intuitive psychology, and a mechanism for categorizing objects into agents and non-agents based on the capacity to act in goal-directed ways (Baillargeon et al., 2010; Barrett et al., 2013; Barrett \& Behne, 2005; Carey, 2009; Spelke, 1994). This is not to say that many or most people do not have afterlife beliefs or endorse some form of mind-body dualism. Explicit beliefs of this sort are perfectly compatible with the absence of continued existence responses in responses to vignettes of the kind used in our study. These varied beliefs and responses are consistent with Harris and Giménez's (2007) proposal that multiple ideologies might exist within the same mind. The "biological" conception of life and mind as ending with the death of the body may be the most common, and possibly universal, conception across cultures, and perhaps the first conception 
that comes to mind when asked questions about a dead person. Explicit religious notions of an afterlife may co-exist with this biological conception in some people, but if intuitive materialism is correct, they might require suppressing the immediate biological response that the person in question is dead and therefore no longer an agent. Religious beliefs about the afterlife, because they are not immediately generated by an evolved cognitive system, may be more ambiguous in the inferences that they generate, which might require a greater degree of conscious deliberation. Examining which afterlife beliefs are relatively easy to entertain and which are more difficult remains an important goal for future research, and one that might be facilitated by exploring the design of the evolved cognitive mechanisms that enable them.

Acknowledgements: We would like to thank the participants at all of our field sites for their generous participation in our study, and the field research assistants who helped collect these valuable data.

Funding: This work was supported by a grant from the United Kingdom's Arts and Humanities Research Council (for the AHRC Culture and the Mind Project) and by the Hang Seng Centre for Cognitive Studies, University of Sheffield.

\section{References}

Astuti, R., \& Harris, P. L. (2008). Understanding mortality and the life of the ancestors in rural Madagascar. Cognitive Science, 32(4), 713-740.

Atkinson, Q. D., \& Bourrat, P. (2011). Beliefs about God, the afterlife and morality support the role of supernatural policing in human cooperation. Evolution and Human Behavior, 32(1), 4149. 
Baillargeon, R., Scott, R. M., \& He, Z. (2010). False-belief understanding in infants. Trends in cognitive sciences, 14(3), 110-118.

Barrett, H. C., \& Behne, T. (2005). Children's understanding of death as the cessation of agency: a test using sleep versus death. Cognition, 96(2), 93-108.

Barrett, H.C., Broesch, T., Scott, R.M., He, Z., Baillargeon, R., Wu, D., Bolz, M., Henrich, J., Setoh, P., Wang, J., \& Laurence, S. (2013). Early false-belief understanding in traditional nonWestern societies. Proceedings of the Royal Society of London, Series B. 280(1755): 20122654.

Bates, D., Maechler, M., Bolker, B., \& Walker, S. (2014). lme4: Linear mixed-effects models using Eigen and S4. R package version 1.1-7, http://CRAN.R-project.org/package=lme4.

Bering, J. (2002). Intuitive conceptions of dead agents' minds: The natural foundations of afterlife beliefs as phenomenological boundary. Journal of Cognition and Culture, 2, 263-308.

Bering, J. and Bjorklund, D. F. (2004). The natural emergence of reasoning about the afterlife as a developmental regularity. Developmental Psychology, 40, 217-233.

Bering, J. M., Hernández Blasi, C., \& Bjorklund, D. F. (2005). The development of afterlife beliefs in religiously and secularly schooled children. British Journal of Developmental Psychology, 23(4), 587-607.

Bloom, P. (2007). Religion is natural. Developmental Science, 10(1), 147-151.

Boyer, P. (2001). Religion explained: The evolutionary origins of religious thought. Basic Books.

Carey, S. (2009). The origin of concepts. New York: Oxford University Press.

Chudek, M., McNamara, R. A., Birch, S., Bloom, P., \& Henrich, J. (2018). Do minds switch bodies? Dualist interpretations across ages and societies. Religion, Brain \& Behavior, 8(4), 354368.

Cohen, E., Burdett, E., Knight, N., \& Barrett, J. (2011). Cross-Cultural similarities and differences in person-body reasoning: Experimental evidence from the United Kingdom and Brazilian Amazon. Cognitive Science, 35(7), 1282-1304.

Harris, P. L., \& Giménez, M. (2005). Children's acceptance of conflicting testimony: The case of death. Journal of Cognition and Culture, 5(1-2), 1-2.

Henrich, J., Heine, S. J., \& Norenzayan, A. (2010). The weirdest people in the world? Behavioral and Brain Sciences, 33(2-3), 61-83.

Hodge, K. M. (2008). Descartes' mistake: How afterlife beliefs challenge the assumption that humans are intuitive Cartesian substance dualists. Journal of Cognition and Culture, 8(3-4), 3-4. 
Keil, F. C. (1989). Concepts, kinds, and cognitive development. Cambridge, MA: MIT Press.

Kuhlmeier, V. A., Bloom, P., \& Wynn, K. (2004). Do 5-month-old infants see humans as material objects? Cognition, 94(1), 95-103.

Leslie, A. M. (1994). ToMM, ToBy, and Agency: Core architecture and domain specificity. In L. A. Hirschfeld \& S. A. Gelman (Eds.), Mapping the mind: Domain specificity in cognition and culture (pp. 119-148). New York, NY: Cambridge University Press.

Matan, A., \& Carey, S. (2001). Developmental changes within the core of artifact concepts.

Cognition, 78(1), 1-26.

McCloskey, M. (1983). Intuitive physics. Scientific American, 248(4), 122-131.

Pew Research Center (2008). U.S. Religious Landscape Survey: Religious Beliefs and Practices. Retrieved from http://www.pewforum.org/2008/06/01/u-s-religious-landscape-survey-religiousbeliefs-and-practices/

Pew Research Center (2012). The Global Religious Landscape. Retrieved from http://www.pewforum.org/2012/12/18/global-religious-landscape-exec/

R Core Team (2014). R: A language and environment for statistical computing. R Foundation for Statistical Computing, Vienna, Austria. URL http://www.R-project.org/.

Spelke, E. (1994). Initial knowledge: Six suggestions. Cognition, 50(1), 431-445.

Sperber, D. (1996). Explaining culture. Oxford: Blackwell Publishers. 